\title{
Operational Definition and Assessment
}

Dr. Robert A Ross, University of Detroit Mercy 


\section{Operational Definition and Assessment}

\section{Introduction}

Do you like assessment? We are glad to hear that because you are going to be doing more of it. Faculty are assessing student learning outcomes, departments are assessing faculty performance and course outcomes, university administrators are assessing departments, and external agencies (private and governmental) are assessing colleges and universities.

As engineering educators we have been assessing student learning and course and program outcomes for years under the context of ABET evaluation, we are much better positioned than some of our liberal arts colleagues. Those of us who teach as part of the engineering curriculum have recognized for many years that if we do not measure what students are learning then we really do not know what or how to teach. We believe that by measuring student learning, with valid and robust instruments, we can adjust the curriculum and pedagogy to increase student learning. We should be teaching with research based active-learning activities, assessing what our students know and address their misunderstandings before they are unsuccessful. Doing this properly should be considered part of our professional responsibility as we endeavor to be more inclusive and attract and retain more women and persons of color into science and engineering.

In this paper we will argue why structuring student learning outcomes around operationally defined technical terms provides a coherent pathway to assessment. We believe the use of the operational definition of technical terms should be an organizing principle in physics and engineering courses. How the careful use of operational definitions can allow students to understand difficult concepts in physics and engineering and to apply them in new and varied contexts. We will provide examples of operationally defined technical terms and their practical use in the classroom and laboratory. We will show that by organizing student learning outcomes and course objectives around the principle of operational definition we can simplify the assessment of student learning, determine student strengths and weaknesses, and develop strategies to increase student achievement.

While the ABET General Criteria 5 on curriculum is silent on calculus-based physics the program criteria are not. The program criteria for Architectural Engineering states; "The program must demonstrate that graduates can apply mathematics through differential equations, calculus-based physics, and chemistry." The program criteria for Mechanical Engineering states "basic science" while Electrical Engineering mentions "physical science." While we are not ABET evaluators, in our experience, all of the engineering students at our institution (we do not have an engineering technology program) are required to take a year of calculus-based physics with the associated laboratories. We will show how we can use the principle of operational definition of fundamental concepts from calculus-derivatives and integrals, and incorporate them into introductory physics courses. 


\section{Operational Definition}

We use the term operational definition in a somewhat loose fashion. We are not trying to demonstrate the existence of the Higgs boson to six standard deviations, rather we are trying to teach undergraduate students the meaning of time, displacement, speed, velocity, acceleration, mass, force, work, energy, charge, conductors, insulators, and complete circuits etc. These are all words and phrases they have heard hundreds of times and yet very few know what they mean. Operational definitions give meaning to terms by describing the process by which they are measured. We also use operational language to describe the process of certain mathematical procedures like integration and differentiation.

We believe in the constructivist approach to learning. Students create their own concepts based upon what they already believe and then subsequently upon what they do. Our operational approach is strongly influenced by the work of Arons ${ }^{1}$ and McDermott. ${ }^{2,3,4}$ We also utilize interactive lecture demonstrations. ${ }^{5}$ We believe the operational process is an intrinsic part of how humans learn. Our evidence for this belief is partly anecdotal; however it did have a powerful effect on teaching and learning at our school.

My first experience with the pedagogical strength of an operational definition was over 20 years ago. My daughter, who is now a mechanical engineer at a Toyota subsidiary, was in pre-school. I chaperoned a class field trip to the Cranbrook Science Center, a well-known, highly respected and exclusive, private institution with a college preparatory K-12 curriculum and a graduate school of design and architecture. There were about two dozen pre-school students, four year olds, who were very excited to hear about dinosaurs.

Our docent had the group in an auditorium and passed around a couple of fossils (most likely replicas) for the students to touch. After a few minutes he asked a seemingly innocuous question - "What is a dinosaur?" Immediately a little boy raised his hand and was called on by the docent, "I don't know." He said, as little boys do. By now, my mind was having quite a bit of trouble wrapping itself around this question. I knew that dinosaurs laid eggs, but they were not reptiles. I thought they were cold blooded, but there was some discussion at the time that TRex was a warm blooded. Actually, I was quite flustered. I really didn't know! A little girl raised her hand, "Dinosaurs are what paleontologists dig up." (She did in fact say paleontologists.) It was like a bulb went off in my head. That statement is a perfectly good operational definition of a dinosaur; at least to a four year old. At the time, it seemed to me that the meaning of the word "dinosaur" was imbedded in the operations performed by the paleontologists - digging. The meaning of the noun comes about from the verbs associated with it. As Bridgman says, "namely that the proper definition of a concept is not in terms of its properties but in terms of actual operations; for of course the true meaning of a term is to be found by observing what a man does with it, not by what he says about it." (or apparently a four year old girl. $)^{6}$

Operational definitions define a process of measurement. We argue, following Bridgeman, that it is this process that gives meaning to technical terms. Furthermore, if the learning outcomes are closely related to operational definitions they become easier to assess and interpreting the assessment data leads to concrete ways to improve curriculum and pedagogy. We believe that if 
students have the opportunity to observe, or better yet perform, a measurement they can develop an understanding that is otherwise very difficult to achieve.

\section{Student learning outcomes assessment}

Several years ago students in the second semester general physics course were asked an openended question on the final exam, "What is electric charge?" The answers ranged from " $a$ fundamental property of matter" to "the energy of the force of the electric field." Of course they could solve all manner of tricky problems involving the calculation of forces and fields but after 15 weeks of instruction the students had no idea what electric charge was. This was not surprising considering the definition in the textbook. The most current edition of Halliday and Resnick says, "Electric charge is an intrinsic property of the fundamental particles that make up objects such as the rods, silk, and fur. That is, charge is a property that comes automatically with those particles wherever they exist."7 It is no wonder students do not understand what charge is, those two sentences do not convey any meaning with regard to determining whether or not an object is electrically charged. From an operational point of view the definition is quite simple. If you bring an object close to an electroscope and the leaf deflects, then the object is electrically charged. There is no ambiguity in this definition. Students can see the leaf deflect when charged rubber and glass rods are brought into close proximity to the ball or plate on the top of an electroscope. The text by Mazur ${ }^{8}$ is one of the few books that use this operational approach to the definition of electric charge.

The strategy we chose to help improve student understanding of these topics was to change some of the second semester introductory physics course learning outcomes. We based the learning outcomes on an operational procedure and base the assessment on an explanation of what one would expect to happen if the experiment were performed or why the apparatus acts the way it does. For simplicity we only show 5 learning outcomes that are operationally based and briefly discuss the assessment of the first two. Many of the assessments used in this work are taken or adapted from Ref 1 .

The student will:

(a) develop operational definitions of electrical charge;

(b) explain the evidence for the existence of only two types of charge;

(c) determine if a material is a conductor, a dielectric, or a photoconductor;

(d) apply Coulomb's law to systems of charged objects;

(e) identify charge transfer mechanisms;...

Examples of the assessment of learning outcome (a) could be:

- What is electric charge? That is, how can you tell if an object is charged?

- How does an electroscope work? A sketch may aid in your explanation.

- Explain what will happen if a negatively charged rubber rod is brought into proximity of an electroscope with excess positive charge? 
Examples of the assessment of learning outcome (b) could be:

- Why do we believe that there are only two types of charge?

- Suppose that you had 3 objects, A, B, and C, that you determined each had electric charge. Furthermore, suppose that the objects were observed to interact with each other according to the diagram shown here. Would this experimental result be significant? Briefly explain your reasoning.

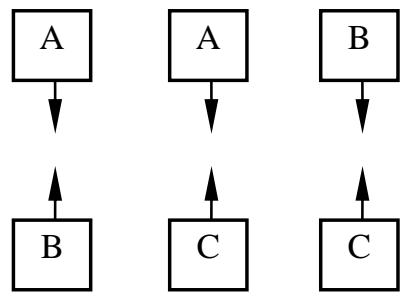

We also use the operational approach in the first semester introductory physics course. We discuss a couple of outcomes and their assessment in the context of doing calculus. In that sense, we treat differentiation and integration as operational processes.

The student will:

(a) develop operational definitions for technical terms;

(b) apply geometrical scaling laws to appropriate physical situations;

(c) compare written, algebraic, and graphical descriptions of motion;

(d) recognize the relationships between $x(t), v(t)$, and $a(t)$ graphs;

Two of the learning outcomes in our first semester physics course are: Compare written, algebraic, and graphical descriptions of motion; recognize the relationships between $x(t), v(t)$, and $a(t)$ graphs. The expectation is that students can describe objects moving in one dimension in terms of position, displacement, speed, velocity, and acceleration. That they know what the graph of an object is that is speeding up, or slowing down, turning around, etc. Students are asked a series of questions related to a set of velocity as a function of time graphs. Correct answers are obtained by following a procedure that requires students to think about the three types of information that can be conveyed in this type of graph. The speed and velocity are ascertained by examining the value of the velocity as time progresses, the displacement is determined from the integral and the acceleration from the slope.

Some sample graphs are shown below in Fig. 1. The graphs vary from term to terms however all of the graphs are comprised of straight line segments. The questions also vary from term to term. One term we may ask, "Which object (or objects) spent some time speeding up?" The next term it may be "Which object (or objects) spent some time slowing down?" We try to maintain a consistent level of difficulty from term to term. The number of question has varied from 7 to 12 .

All of the students have successfully completed the requirements for the introductory calculus course at the university either with a minimum grade of $\mathrm{C}$ or through a placement exam. It seems reasonable to expect that they know the Fundamental Theorem of Calculus which states that $\int_{a}^{b} f(x) d x$ is the area under the $f(x)$ graph from $a$ to $b$ and also that the slope of the tangent line represents the derivative. ${ }^{9}$ The results of assessment seem to show otherwise. A large percentage of students cannot apply these calculus concepts to functions when they are shown graphical representations. 
Examples of the assessment of learning outcomes (c) and (d) are:

Shown below are six velocity versus clock reading histories which describe the one dimensional motion of six objects that started out from the origin $x=0 \mathrm{~m}$ at time $t=0 \mathrm{~s}$.

(a) Which object (or objects) are located at the origin, $x=0$, at the clock reading $t=2 \mathrm{~s}$ ?

(b) Which object (or objects) spends at least some time moving in the positive direction?

(c) Which object (or objects) moves with a constant, nonzero acceleration during the entire time interval?

(d) Which object (or objects) exhibited positive acceleration sometime during the given period?

(e) Which object (or objects) changed direction?

(f) Which object (or objects) was at rest for some period of time?
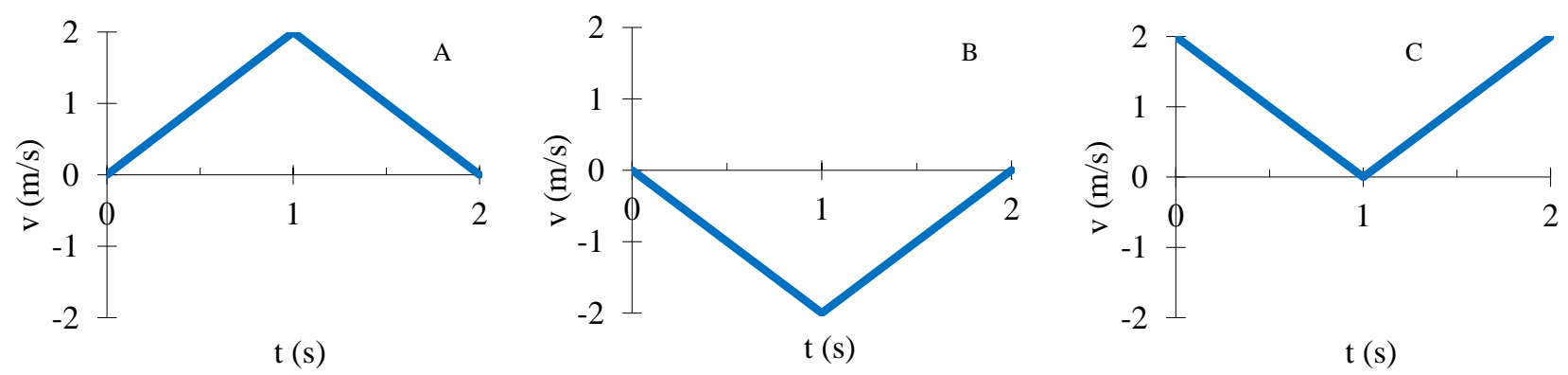

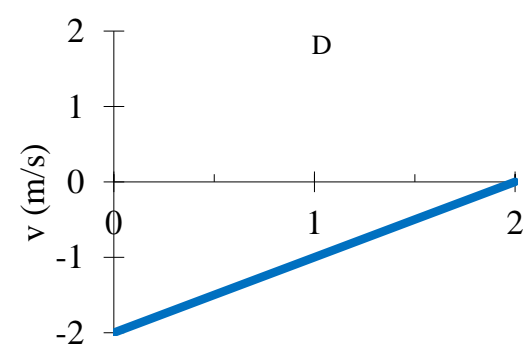

$\mathrm{t}(\mathrm{s})$

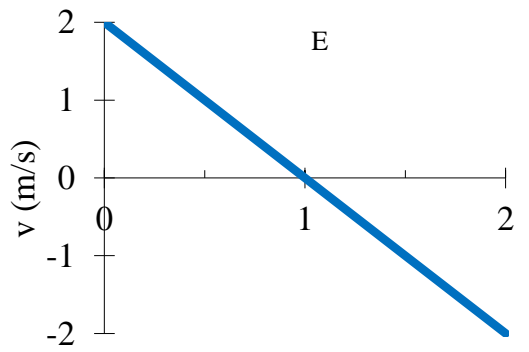

$\mathrm{t}(\mathrm{s})$

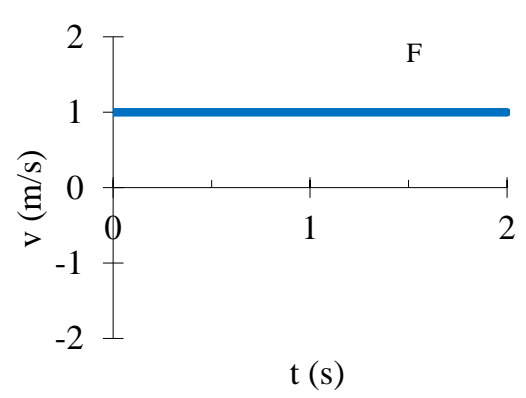

Figure 1

Sample velocity versus time graphs used in the assessment of calculus learning outcomes.

In Fig. 2 we show the results of this assessment. Since the number of questions asked varied from term to term the percentage of correct responses was placed in bins $20 \%$ wide. The results for eight sections of introductory physics classes over the past 6 years are shown. The average number of correct responses is $37 \%$ and the total number of students assessed is 136 . The results that are shown include a few students that repeated the course and some that dropped after taking the test. Six students were assessed twice, they repeated the course, and one student was assessed three times. Their results are shown below in Table 1 . Their scores typically improve as one would expect. 


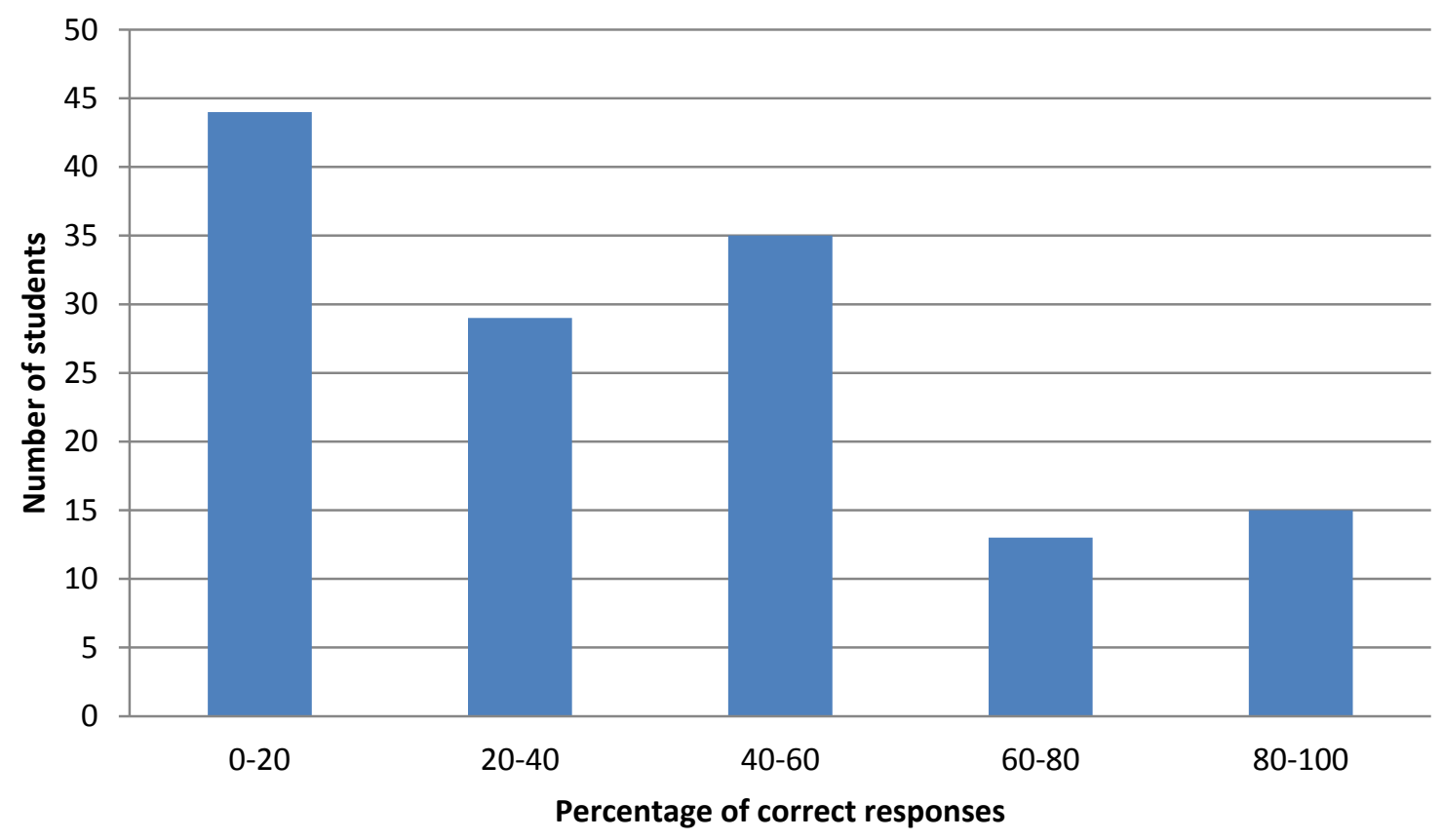

Figure 2

Number of students with the corresponding percentage of correct responses in $20 \%$ bins.

\begin{tabular}{|c|c|c|c|}
\hline Student & $1^{\text {st }}$ & $2^{\text {nd }}$ & $3^{\text {rd }}$ \\
\hline $\mathrm{a}$ & $8 \%$ & $83 \%$ & \\
\hline $\mathrm{b}$ & $25 \%$ & $42 \%$ & $71 \%$ \\
\hline $\mathrm{c}$ & $29 \%$ & $58 \%$ & \\
\hline $\mathrm{d}$ & $50 \%$ & $50 \%$ & \\
\hline $\mathrm{e}$ & $0 \%$ & $8 \%$ & \\
\hline $\mathrm{f}$ & $0 \%$ & $25 \%$ & \\
\hline
\end{tabular}

Table I

Percentage of correct responses for students that repeated the assessment.

As a result of this assessment, we intend to introduce an additional collaborative activity into the curriculum. Students will work in small groups of 2 or 3 students and generate graphs of a ball bouncing on a horizontal surface. They are to assume that the ball is dropped from a height of $1.25 \mathrm{~m}$, that $g=10 \mathrm{~m} / \mathrm{s}^{2}$, it rebounds to its original height, and subsequently impacts 3 times. They are to generate $x(t), v(t)$, and $a(t)$ graphs of this motion and to explain their reasoning. This is an open-ended problem in the sense that they can easily calculate the time and speed of the ball upon impact, but the rebound time is ill defined. Hopefully it will lead to better understanding of motion under constant acceleration conditions. 


\section{Conclusion}

The assessment of student learning is becoming more and more important. It is likely that we will all be doing more of it in the future. We believe that by utilizing operational definitions in student learning outcomes we can more readily generate valid assessments. At the same time we can use the assessment to change instructional practice so that more students are able to achieve at the levels we want.

\section{References}

${ }^{1}$ A. Arons, Teaching Introductory Physics, John Wiley \& Sons, New York, 1996.

${ }^{2}$ L.C. McDermott and E.F, Redish. "Resource Letter: PER-1: Physics Education Research", Am. J. Phys., 67 (9), September 1999, pp. 755-767, and references therein.

${ }^{3}$ L.C. McDermott, P.S. Shaffer, and the Physics Education Group, Tutorials in Introductory Physics, Prentice Hall, 1998.

${ }^{4}$ L.C. McDermott, et.al., Physics by Inquiry, John Wiley \& Sons, 1996.

${ }^{5}$ D.R. Sokoloff, and R. Thornton. Interactive lecture demonstrations: active learning in introductory physics, John Wiley \& Sons, 2004.

${ }^{6}$ P.W.Bridgman, The Logic of Modern Physics, The Macmillian Company, New York, 1928, pg. 6-7.

${ }^{7}$ D. Halliday, R. Resnick, and J. Walker. Fundamentals of Physics, John Wiley \& Sons, New York, $10^{\text {th }}$ edition extended, 2015, pg 611.

${ }^{8}$ E. Mazur, Principles \& Practice of Physics, Pearson, 2015.

${ }^{9}$ H.Anton, I. Bivens, and S. Davis, Calculus Early Transcendentals Single Variable, $9^{\text {th }}$ ed, John Wiley \& Sons, 2009, pg 362. 